\title{
Schriftenreihe des Bundesinstituts für Sportwissenschaft
}

Band 37 Prof. Dr. Kurt-Alphons Jochheim / Prof. Dr. Paul van der Schoot

\section{Behindertensport und Rehabilitation}

Ergebnisse eines Forschungsprojekts des Instituts für Rehabilitation und Behindertensport der Deutschen Sporthochschule Köln

Teil I: Dokumentation und Bericht zum Stand der Forschung im Bereich des Sports mit psychisch Behinderten und geistig Retardierten

1981. DIN A 5, 468 Seiten, ISBN 3-7780-7381-8 (Bestellnummer 738)

Band 38 Dr. Jürgen Innenmoser / Prof. Dr. Heinz Kosel

\section{Behindertensport und Rehabilitation}

Ergebnisse eines Forschungsprojekts des Instituts für Rehabilitation und Behindertensport der Deutschen Sporthochschule Köln

Teil II: Dokumentation und Bericht zum Stand der Forschung im Bereich des Sports mit Körperbehinderten, Hörgeschädigten, Sehgeschädigten

1981. DIN A 5, ISBN 3-7780-7391-5 (Bestellnummer 739)

In 2 Bänden werden die Ergebnisse einer systematischen Analyse der deutsch- und zum größten Teil auch englischsprachigen Untersuchungen zum Behindertensport vorgestellt. Daneben werden auch die Methoden dieser Untersuchungen diskutiert. Im Mittelpunkt stehen Forschungen zu den Bereichen „Sport mit Psychisch Behinderten“, „Sport mit Geistig Retardierten“, „Sport mit Körperbehinderten“, „Sport mit Hörgeschädigten“, „Sport mit Sehgeschädigten“. In Ergänzung dazu wird der Bereich des Schulsonderturnens sowie des Therapeutischen Reitens dokumentiert. Den SchluB bildet die Darstellung der Ausbildungssituation. Alle Kapitel enden mit einer Empfehlung für zukunftige Forschung.

\section{Texte - Quellen - Dokumente zur Sportwissenschaft}

Band 15 Prof. Dr. Heinz Denk / Prof. Dr. Gerhard Hecker

\section{Texte zur Sportpädagogik, Teil I}

Dieser Band bietet einen Längsschnitt über sportpädagogisches Denken von Platon und Aristoteles bis Coubertin. Es sind Texte von Autoren aufgenommen worden, die überzeitliche Wirkung hatten und Grundlagen für eine Problemgeschichte der Sportdidaktik anbieten. Dabei sind oft überraschend aktuelle Themen angesprochen.

1981. DIN A 5, 296 Seiten, ISBN 3-7780-6151-8 (Bestellnummer 615)

\section{S P ORTW IS SEN S C HAFT 11 . Jahrgang $1981 / 3$}

Herausgegeben vom Deutschen Sportbund, vom AusschuB Deutscher Leibeserzieher und vom Bundesinstitut für Sportwissenschaft.

(C) 1981 by Verlag Karl Hofmann, 7060 Schorndorf. Alle Rechte, auch das der Übersetzung in fremde Sprachen, vorbehalten. Nachdruck und Vervielfältigungen jeder Art nur mit Genehmigung des Verlags.

Beilagenhinweis: Einer Teilauflage dieser Ausgabe liegt der Prospekt „Sport im Winterhalbjahr" des Hofmann-Verlags bei. 